\title{
石墨烯增强铜基复合材料的研究进展
}

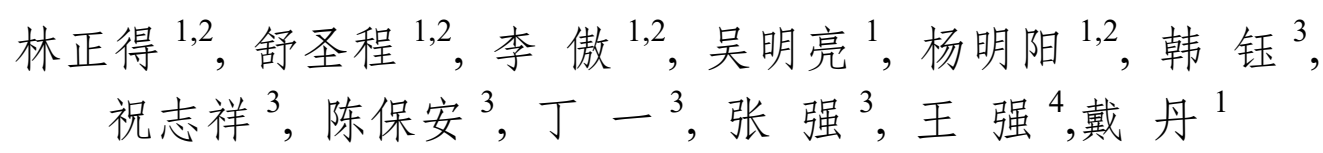

(1. 中国科学院 宁波材料技术与工程研究所 表面工程事业部, 宁波 $315201 ; 2$. 中国科学院大学, 北京 100049; 3. 全球能源互联网研究院有限公司先进输电技术国家重点实验室, 北京 102209; 4. 国网山西省电力公司, 太原 030001)

摘 要: 石墨烯具有超高的比表面积和优异的力学性能，是铜基复合材料理想的增强体。传统的粉末治金工艺很难 解决石墨烯在铜基体中的分散问题，以及石墨烯与铜基体结合性差的难题。随着近些年研究者对石墨烯一铜界面问 题深入的探索，一些新的制备工艺不断出现。本文系统地介绍和对比了近几年石墨烯增强铜基复合材料的制备工艺， 概述了关于石墨烯/铜复合材料力学性能的研究进展, 总结了石墨烯增强铜基复合材料力学性能的机理, 并对未来 石墨烯增强铜基复合材料的研究重点进行了展望。

关 键 词: 石墨烯; 铜基复合材料; 力学性能; 增强机理; 综述

中图分类号: TG146 文献标识码: A

\section{Preparation and Mechanical Property of Graphene-reinforced Copper Matrix Composites}

\author{
LIN Zheng-De ${ }^{1,2}$, SHU Sheng-Cheng ${ }^{1,2}$, LI Ao ${ }^{1,2}$, WU Ming-Liang ${ }^{1}$, YANG Ming-Yang ${ }^{1,2}$, HAN Yu ${ }^{3}$, \\ ZHU Zhi-Xiang ${ }^{3}$, CHEN Bao-An ${ }^{3}$, DING Yi ${ }^{3}$, ZHANG Qiang ${ }^{3}$, WANG Qiang ${ }^{4}$, DAI Dan ${ }^{1}$
}

(1. Division of Surface Engineering and Remanufacturing, Ningbo Institute of Industrial Technology, Chinese Academy of Sciences, Ningbo 315201, China; 2. University of Chinese Academy of Sciences, Beijing 100049, China; 3. State Key Laboratory of Advanced Transmission Technology, Global Energy Interconnection Research Institute Co., Ltd., Beijing 102209, China; 4. State Grid Shanxi Electric Power Company, Taiyuan 030001, China)

\begin{abstract}
Graphene, which has two-dimensional carbon single atomic layer, attracts great attention due to its superb mechanical, electrical and thermal properties. In addition to its excellent mechanical properties, a large surface area (about $2600 \mathrm{~m}^{2} \cdot \mathrm{g}^{-1}$ ) makes it an ideal reinforcement for copper-based composites. However, graphene owns a low density $\left(2.2 \mathrm{~g} \cdot \mathrm{cm}^{-3}\right)$, while the density of copper is about $8.9 \mathrm{~g} \cdot \mathrm{cm}^{-3}$. The traditional powder metallurgy process is difficult to solve the problem of uniform dispersion of graphene in the copper matrix and the poor bonding strength between graphene and copper due to the huge density difference between copper and graphene. With the in-depth exploration in the issue of graphene/copper interface in recent years, some novel preparation processes and strengthening mechanisms were proposed and demonstrated. This review systematically introduces and compares the recently-developed preparation processes of graphene-reinforced copper composites, also summarizes the mechanism of mechanical enhancement in graphene-reinforced copper matrix composites.
\end{abstract}

Key words: graphene; copper matrix composites; mechanical property; strengthening mechanism; review 
铜及铜合金具有优异的导电导热性能, 良好的 塑性、韧性与延展性, 广泛应用于电子电气行业、 机械制造业等领域，在现代工业体系中占有重要位 置。但是传统的铜及铜合金材料存在强度低、高温 性能差等缺点 ${ }^{[1]}$, 限制了铜及铜合金的进一步应用。 随着现代工业技术的高速发展，对铜及铜合金的力 学性能提出了更高要求。如何在铜及铜合金中引入 合适的增强相制备高性能的铜基复合材料, 以及如 何更好地发挥基体与增强相的协同作用, 成为研究 者关注的热点问题 ${ }^{[2-5]}$ 。

在关于铜基复合材料的研究中，多采用合金元 素(如 $\mathrm{Ti} 、 \mathrm{~W} 、 \mathrm{Ni}$ 等元素 $)^{[6-8] \text { 、碳纤维 }}{ }^{[9-10]}$ 、碳纳米 管 ${ }^{[5,11-12]}$ 等作为增强相提升铜基复合材料的性能。使 用合金元素作为增强相，可以显著提高铜基复合材 料的力学性能, 但是会大幅度降低材料的导电、导 热性能 ${ }^{[7-8]}$; 使用碳纤维作为增强相制备的铜基复 合材料, 具有高导热、高导电性能以及优异的耐磨 损性能, 但是由于碳纤维与铜基体的界面润湿性差, 使得碳纤维增强铜基复合材料的力学性能有大幅度 的降低 ${ }^{[5,10]}$ 。碳纳米管对铜基体的力学性能有小幅 度提高, 但是碳纳米管制备难度大, 成本高, 碳纳 米管在铜基体中易团聚。而且在碳纳米管增强铜基 复合材料的拉伸断面中经常发现大量处于拔出脱落 状态的碳纳米管 ${ }^{[11]}$, 说明碳纳米管与铜基体的结合 状态不够牢固, 界面结合仍需进一步增强。因此, 选 用新型增强相材料是提高铜基复合材料性能的关键。

石墨烯的发现 ${ }^{[13]}$ 与大规模制备 ${ }^{[14]}$ 为提高铜基 复合材料的性能提供了新的解决方法。石墨烯是一 种单层碳原子构成的二维纳米材料, 具有优异的力

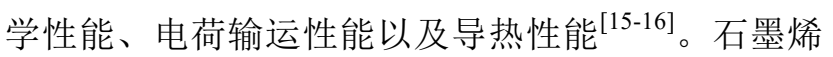
作为铜基复合材料的增强相有其独特的优势，例如， 化学稳定性 ${ }^{[17]}$ 、高强度和刚度 ${ }^{[18]}$ 、优越的导电性能 和导热性能 ${ }^{[16]}$ 。但是, 由于石墨烯密度小、分散性 差 $^{[19]}$ 、与熔融铜界面张力不同以及界面结合问题 ${ }^{[20-21]}$,
很难实现石墨烯在铜基材料中均匀分散以及石墨烯 与铜的强界面结合。近年来, 石墨烯增强铜基复合 材料的新工艺不断出现，本文将从石墨烯增强铜基 复合材料的制备方法、力学性能等方面进行详尽总 结，并对其未来发展趋势进行展望。

\section{1 石墨烯增强铜基复合材料的制备方 法及力学性能}

如何实现石墨烯在铜基体中的均匀分散，形成 强界面结合以及维持结构的稳定性，是制备石墨烯 增强铜基复合材料的关键问题，选择合适的制备方 法尤为重要, 目前, 主要有粉末治金法(Powder Metallurgy, PM) ${ }^{[22-23]}$ 、化学气相沉积法 (Chemical

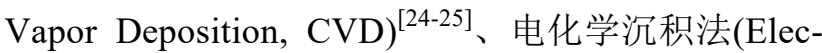

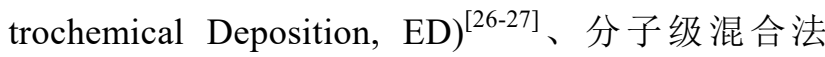
(Molecular-level Mixing, MLM) ${ }^{[28-30]}$ 等制备方法。

表 1 为各种制备工艺的技术路线及其优缺点。

\section{1 粉末治金法}

粉末冶金法是指将增强相与金属粉末混合后采 用模压或等静压预压成型，通过热压烧结(Hotpressing, HP)、放电等离子烧结(Spark Plasma Sintering, SPS)或等离子活化烧结 (Plasma Activated Sintering, PAS)得到金属基复合材料的一种传统方 法 ${ }^{[31]}$, 也是目前较为成熟，应用最为广泛的金属基 材料制备工艺。为了使烧结体致密化，研究者还会 选用热轧 ${ }^{[32]}$ 、热挤压 ${ }^{[33]}$ 等二次机械加工工艺对烧结 体进行进一步处理。

Ponraj 等 ${ }^{[22]}$ 使用聚乙烯醇(PVA)对球型铜粉进 行亲水化处理, 然后将氧化石墨烯(GO)水溶液与经 PVA 处理的球型铜粉进行机械混合, 对得到的 Cu-PVA-GO复合浆料进行高温热处理，再对得到铜 /石墨烯复合粉末进行冷压成型得到铜/石墨烯复合 块体材料。当石墨烯添加量为 $2 \mathrm{wt} \%$ 时，复合材料的

表 1 为各种制备工艺的技术路线及其优缺点

Table 1 Processing cessing routes and merits/demerits of processing techniques

\begin{tabular}{|c|c|c|}
\hline Processing technique & Processing route & Merits/Demerits \\
\hline PM & Ball milling (ultrasonication) + Hot pressing & $\begin{array}{l}\text { Excellent dispersion, good mechanical bonding/higher de- } \\
\text { fect concentration }\end{array}$ \\
\hline CVD & Ball milling + CVD + Hot pressing & $\begin{array}{l}\text { Random distribution, in-situ grown grapheme with perfect } \\
\text { quality, excellent interfacial bonding/grain growth, low } \\
\text { graphene integrity }\end{array}$ \\
\hline ED & Pulse reverse electrodeposition + Annealing & Smooth, highly dense, uniform dispersion, fine grain size \\
\hline MLM & Molecular Level Mixing + SPS & $\begin{array}{l}\text { Homogeneous dispersion, low defect density after reduc- } \\
\text { tion, strong interactions between } \mathrm{Cu} \text { and graphene }\end{array}$ \\
\hline ARB & Accumulative roll bonding + Hot compaction & $\begin{array}{l}\text { Enhanced interfacial bonding, significant grain refinement/ } \\
\text { poor plasticity }\end{array}$ \\
\hline
\end{tabular}


抗压强度达到 $234 \mathrm{MPa}$, 相比纯铜样品(190 MPa)提 高 $23 \%$, 硬度达到 $75 \mathrm{HRB}$, 相对于纯铜提升 $25 \%$ 。 氧化石墨烯由于良好的分散性而在粉末治金法中被 广泛使用，但是其后期还原过程难以控制，且还原 后石墨烯的结构与质量难以精确调控, 从而对石墨 烯增强铜基复合材料的性能产生不利影响 ${ }^{[22]}$ 。

$\mathrm{Li}$ 等 ${ }^{[23]}$ 通过超声处理悬浮在丙酮中的石墨烯 片, 使石墨烯在丙酮中分散均匀后加入电解铜粉, 经磁力搅拌均匀后真空干燥并进行热压成型, 流程 图如图 1 所示。当添加的石墨烯体积分数为 $2.5 \%$ 时, 复合材料的抗弯强度达到 $441 \mathrm{MPa}$, 维氏硬度为 27.8。由于石墨烯与铜基材料机械混合, 石墨烯分散 不均匀, 石墨烯容易团聚。而且, 石墨烯与铜基体表 面结合形式为范德华力, 结合强度较低, 最终导致 粉末冶金法制备的石墨烯增强铜基复合材料的综合 性能与理论相差较大 ${ }^{[21,34-35]}$ 。

用粉末冶金法制备的铜石墨烯块体样品, 石墨 烯可以作为一种二维屏障, 在晶界处限制位错的运 动, 从而对力学性能产生积极的影响。但是, 增加石 墨烯的添加量时, 很难避免石墨烯的团聚, 从而对 复合材料的力学性能产生负面影响 ${ }^{[36]}$ 。Chu 等 ${ }^{[37]}$ 发 现，添加石墨烯会抑制热压过程中铜晶粒的长大， 从而达到细晶强化的目的, 与纯铜相比, 屈服强度 提升达到 $114 \%$ ，杨氏模量提高 34\%。

\section{2 化学气相沉积法}

化学气相沉积法通常以甲烷等含碳有机物 ${ }^{[38-39]}$ 为碳源, 以金属基体 $(\mathrm{Cu} \text { 或 } \mathrm{Ni})^{[40]}$ 为催化剂, 在金属 基体表面催化生长石墨烯，制备流程如图 2 所示。 与粉末治金法相比, 该法工艺复杂, 但具有过程可 控、石墨烯与铜基体界面结合良好等优点 ${ }^{[25]}$ 。Rho 等 ${ }^{[24]}$ 使用甲烷作为碳源, 在平均粒径为 $5 \mu \mathrm{m}$ 的铜 粉表面通过 CVD 工艺生长石墨烯, 得到了一种三

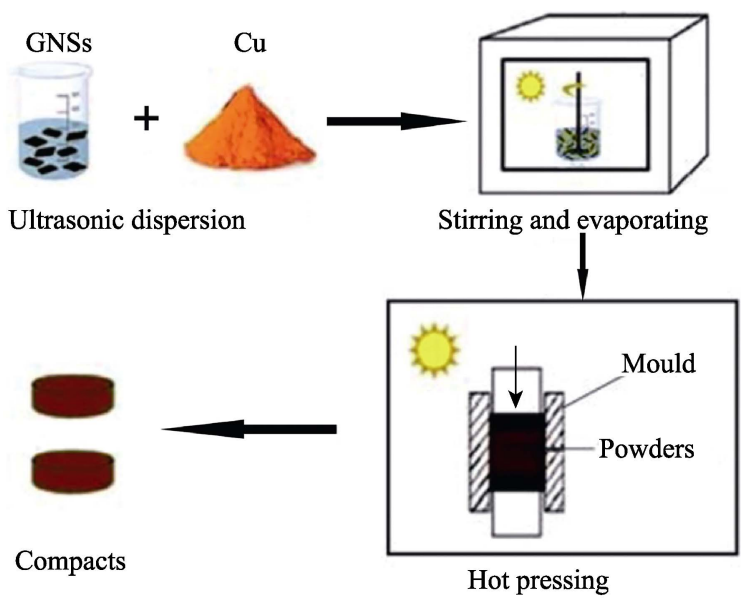

图 1 粉末冶金法制备石墨烯增强铜基复合材料的流程图 ${ }^{[23]}$

Fig. 1 Schematic diagram of PM process of composites ${ }^{[23]}$

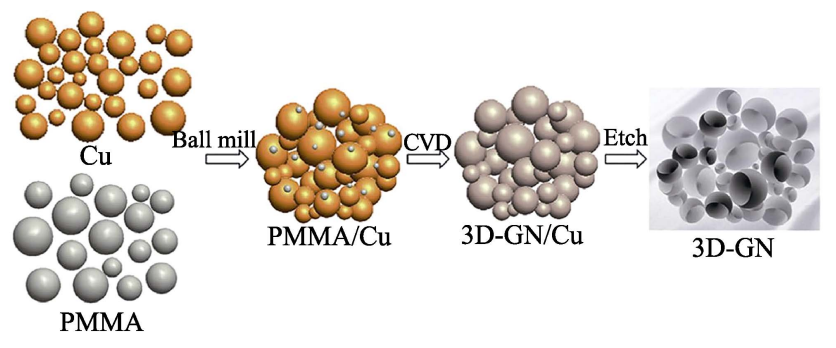

图 2 化学气相沉积法制备石墨烯增强铜基复合材料的示意 图 $^{[25]}$

Fig. 2 Schematic diagram of CVD process of composites ${ }^{[25]}$

维多孔石墨烯铜复合材料, 孔隙率达到 35\%, 在电 极材料与导热材料中有广泛的应用前景。

Chen 等 ${ }^{[25]}$ 采用球磨法(Ball Milling, BM) 将 PMMA 与铜粉均匀混合, 以 PMMA 作为固态碳源, 在铜粉表面通过 CVD 工艺生长石墨烯, 经真空热压 烧结得到石墨烯铜块体材料。这种石墨烯铜复合块体, 石墨烯含量只有 $0.5 \mathrm{wt} \%$, 屈服强度却达到 $290 \mathrm{MPa}$, 约为纯铜的 2.3 倍, 抗拉强度达到 $308 \mathrm{MPa}$, 约为纯 铜的 1.36 倍。在铜粉表面通过 CVD 法生长石墨烯, 可实现石墨烯与铜的均匀混合，与粉末治金法相比 较, 石墨烯与铜粉表面结合紧密, 石墨烯的存在有 效阻碍了铜晶粒中位错的运动, 因而力学性能提升 幅度较大。此外, 通过调控 CVD 工艺参数可对石墨 烯层数进行精确控制 ${ }^{[41-42]}$ 。但是, CVD 法也存在石 墨烯层易磨损、高温下铜晶粒长大的问题。

\section{3 电化学沉积法}

电化学沉积法是采用脉冲交流电源或直流电源 使镀液中的金属离子还原并沉积在基体表面的化学 还原过程。Pavithra 等 ${ }^{[26]}$ 使用脉冲交流电化学沉积 法，硫酸铜溶液作为镀液，制备出了硬度极高 ( 205 GPa) 的石墨烯铜复合薄片, 弹性模量可达 $\sim 137 \mathrm{GPa}$ 。图 3 为电化学沉积示意图, 先通过电化 学剥离法制备表面带有大量官能团的氧化石墨烯,

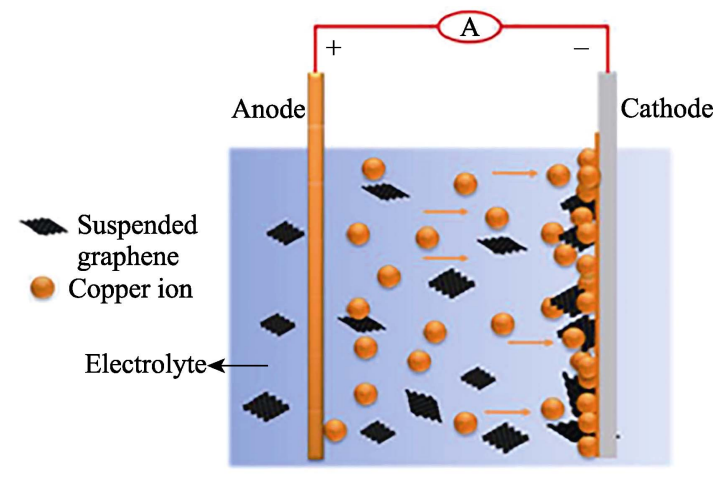

图 3 电化学沉积法制备石墨烯增强铜基复合材料示意图 ${ }^{[26]}$ Fig. 3 Schematic diagram of electrochemcal deposition process of composites ${ }^{[26]}$ 
然后在室温下使用超声波将制备的氧化石墨烯均匀 地分散在硫酸铜电解液中。水解后的氧化石墨烯带 负电, 随着脉冲电流周期性地靠近和远离铜电极保 证了氧化石墨烯在铜基体的均匀分布。电化学沉积 法可以通过调控电流强度、持续时间和脉冲周期等 参数, 对得到的复合材料性能进行调控。而且, 该方 法制备的复合材料中石墨烯分散均匀，有效抑制了 石墨烯的团聚，电性能可达到与纯铜相当的水平; 该方法制备的石墨烯/铜强度范围提升至 2.1 $2.5 \mathrm{GPa}$, 与用该方法处理的纯铜(1.4 1.6 GPa)相比, 强度增强了 $96 \%$; 同时, 该法制备的石墨烯/铜的弹性 模量为 127 137 GPa, 比起该法制得的铜片( 116 GPa) 有所提升 ${ }^{[26]}$ 。与 CVD 工艺相比, 电化学沉积反应温 度较低, 避免了 CVD 过程中晶粒长大的问题。

\section{4 分子级混合法}

分子级混合法是通过 Hummers 法制备氧化石 墨烯(GO), 将氧化石墨烯均匀分散于 $\mathrm{Cu}^{2+}$ 溶液中, $\mathrm{Cu}^{2+}$ 与 $\mathrm{GO}$ 的含氧官能团之间形成化学键, 加入氢 氧化钠溶液形成 $\mathrm{CuO} / \mathrm{GO}$, 然后使用氢气还原 $\mathrm{CuO} /$ $\mathrm{GO}$ 形成 $\mathrm{Cu} / \mathrm{RGO}$ 粉末 ${ }^{[28]}$, 图 4 为分子级混合制备 石墨烯增强铜基复合材料流程。最后通过等离子放 电烧结得到石墨烯铜复合块体材料。Hwang 等 ${ }^{[28]}$ 采用分子级混合法与 SPS 烧结制备的铜石墨烯 复合材料的屈服强度达到 $284 \mathrm{MPa}$, 弹性模量为 $131 \mathrm{GPa}$, 分别比纯铜提高了 $80 \%$ 和 $30 \%$ 。这是因为 分子级混合法基于溶液混合，金属离子更均匀地吸 附于石墨烯薄片表面, 有效抑制了石墨烯团聚, 石墨烯分散的均匀性远远优于直接混合与球磨混 合 ${ }^{[28]}$; 放电等离子烧结可以较好地改善石墨烯与
铜的接触界面，石墨烯与铜结合强度更高 ${ }^{[43]}$, 从而 得到高性能的石墨烯铜复合材料。

\section{5 累积叠轧焊}

有研究者采用累积叠轧焊 (Accumulative Roll Bonding, ARB)的方法提升石墨烯铜复合材料的力 学性能 ${ }^{[44-45]}$ 。ARB 工艺是先制得分散均匀的石墨烯 纳米片悬浮液, 然后通过喷枪将悬浮液均匀地喷涂 在表面光滑且无氧的两块铜条上。再通过辊轧机将 两块铜条叠轧在一起之后, 再将制得的样品切成两 块大小相似的铜板, 然后重复上述步骤 5 6 次后就 得到了石墨烯铜复合材料。Liu 等 ${ }^{[4]}$ 采用该方法将 石墨烯铜复合材料的抗拉强度提升至 $496 \mathrm{MPa}$ ，比 普通纯铜样品提升了 $275 \mathrm{MPa}$, 这主要是由于加工 过程中的应变硬化以及细晶强化。但是该方法会导 致材料的塑性大幅度降低，仅为普通铜样品的 $10 \%$ 。表 2 为石墨烯材料增强铜基材料的力学性能 的相关数据。

\section{2 石墨烯增强铜基材料的技术难点与 机理分析}

\section{1 石墨烯的分散性}

实现石墨烯在铜基体中的均匀分布是提高石墨 烯增强铜基复合材料力学性能的主要因素 ${ }^{[46]}$ 。相反 地，石墨烯在铜基体的团聚则会大大降低复合材料 的力学强度。因此，石墨烯均匀分散于基体中是获 得具有理想力学性能的复合材料的前提。为解决石 墨烯含量较高和分散程度较高时团聚的问题, 研究 人员采取了很多不同的策略, 包括改变加工路线 ${ }^{[47-48]}$,
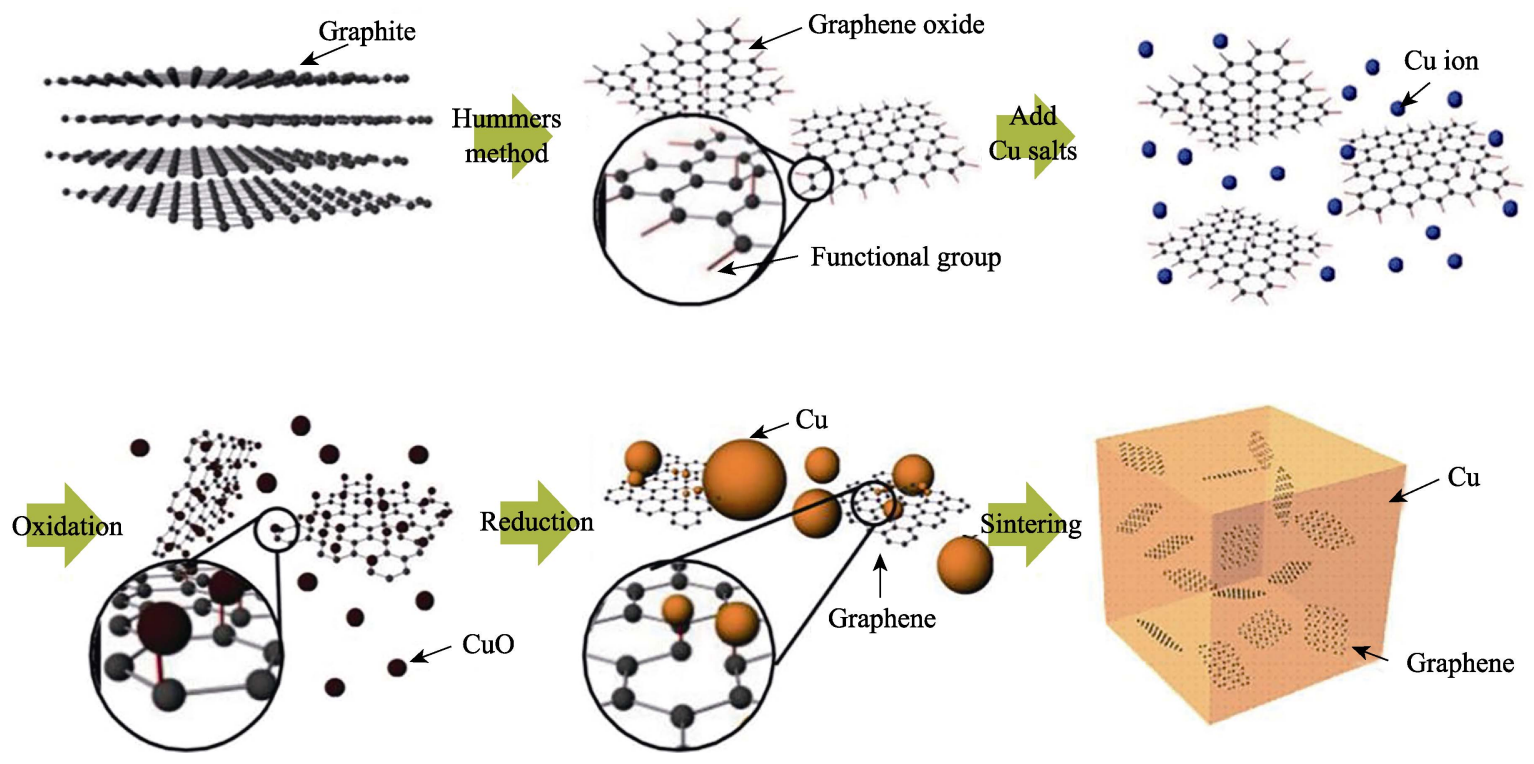

图 4 分子级混合法制备石墨烯增强铜基复合材料示意图 [28]

Fig. 4 Schematic diagram of MLM process of composites ${ }^{[28]}$ 
表 2 石墨烯增强铜基复合材料的力学性能

Table 2 Mechanical properties of graphene-reinforced copper matrix composites

\begin{tabular}{cccccccc}
\hline \multirow{2}{*}{ Researchers } & Processing route & \multicolumn{2}{c}{ Graphene content } & Yield & Tensile & $\begin{array}{c}\text { Compression } \\
\text { strength/MPa }\end{array}$ & $\begin{array}{c}\text { Bending } \\
\text { strength/MPa }\end{array}$ \\
\cline { 3 - 6 } strength/MPa
\end{tabular}

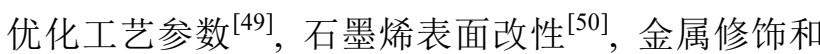
表面覆盖(Ni 等金属)等 ${ }^{[8]}$ 。如果按分散介质来划分, 石墨烯的分散方法主要有固相分散、液相分散以及 固液相结合的分散方法。固相分散中，粉末冶金是 一种能够高效率地使石墨烯均匀分散在金属基粉末 中的方法，它是通过高能混合降低石墨烯与铜粉表 面能的差异，从而抑制了石墨烯的团聚。但同时机 械混合会在混合过程中破坏石墨烯的完整度，引入 大量的缺陷，从而降低了石墨烯对于基体的强化效 率。液相分散则是利用高能超声将石墨烯分散在水、 乙醇等溶液中, 之后再与铜粉混合。但高能超声波 处理不可避免地也会导致缺陷的形成，所以要限制 超声处理的时间。为了取得更好的分散效果，研究 人员将固相分散和液相分散相结合，先通过超声波 将石墨烯分散在溶液中，然后通过球磨将石墨烯和 铜粉混合。这种固液相结合的方法不仅保证了石墨 烯的均匀分散, 而且增强了石墨烯与铜粉的结合, 从而强化了增强效率。

\section{2 石墨烯的润湿性与形成强界面结合}

当外加应力能够有效地传递到铜基体中的石墨 烯上时, 石墨烯增强铜基复合材料的力学强度就会 增强，这要求石墨烯和铜基体之间有强的界面结 合 ${ }^{[6]}$ 。强的界面结合保证了适当的载荷转移，弱的界 面结合会导致受力时裂纹的产生和复合材料的提前 失效。Bartolucci 等 ${ }^{[51]}$ 认为石墨烯金属基复合材料 在热压过程中缺陷处会形成碳化物界面，从而降低 石墨烯对基体的强化效率。为了改善石墨烯与金属 基体的结合，许多研究使用金属离子修饰的还原氧 化石墨烯作为增强相。使用金属离子修饰的还原氧
化石墨烯代替石墨烯纳米片来增加铜基体的强度, 一方面是由于金属离子的存在使得还原氧化石墨烯 不容易团聚，另一方面金属离子会增强还原氧化石 墨烯与铜基体的键合界面，这显著增加了复合材料 的强度。除此之外, 研究人员通过使用氧化石墨烯 还获得了界面良好的石墨烯金属基复合材料 ${ }^{[52]}$ 。 Peng 等 ${ }^{[53]}$ 认为铜基和氧化石墨烯的还原所产生的 残留氧基可以形成强大的共价键，从而显著提高纳 米复合材料的强度。

研究人员在制备石墨烯/铜复合材料时，尤其是 在与金属粉末机械混合和高温烧结过程中，非常注 意保持石墨烯独特的晶格结构与缺陷浓度。这是由 于石墨烯所有的优异性能都来自于其独特的结构, 任何结构的缺陷都会影响到石墨烯作为增强体的力 学增强效果 ${ }^{[54]}$ 。另外, 缺陷的产生还会引入空位, 从而发生化学反应导致石墨烯增强效果的大幅下 降。Cui 等 ${ }^{[5]}$ 研究发现石墨烯结构中缺陷的增多会 降低其负载能力，并严重地影响石墨烯/铜复合材料 的力学强度。研究人员试图通过工艺设计以及优化 工艺参数来解决此类问题。 $\mathrm{Li}$ 等 ${ }^{[56]}$ 采用一步热反应 和 SPS 工艺可以在制备复合材料的过程中很好地保 证石墨烯结构的完整度。

\section{3 石墨烯增强铜基复合材料的强韧化}

由于石墨烯固有的优异力学性质, 研究人员通 过不同工艺制备的石墨烯铜基复合材料的强度得到 非常明显的提高。但是随着金属材料强度的提高, 塑性和韧性通常会下降，强度一韧性呈明显的倒置 关系。在石墨烯铜基复合材料中，石墨烯作为增强 相使得铜基体的强度显著增大，但是其韧性和延展 
性却大大降低，这也是制约石墨烯铜基复合材料实 际工业应用的主要瓶颈之一。近年来，层状结构由 于构型相对简单、制备方法多样且能实现金属基复 合材料性能指标的最优化配置而备受关注。其中, Xiong 等 ${ }^{[57]}$ 受到仿生贝壳结构启发, 通过化学途经 复制冷杉木结构先制备出层状多孔铜结构, 然后浸 渗氧化石墨烯溶液, 制备出还原氧化石墨烯铜基复 合层状材料, 展示出高强度和高韧性, 如图 5 所示。

除此之外, Zhang 等 ${ }^{[58]}$ 通过原位合成法制备了
一种具有三维石墨烯网格/铜构型(3D GN@Cu@Cu) 的石墨烯增强铜基复合材料。该工艺首先在原位上 合成三维石墨烯网格/铜粉末(3D GN@Cu), 使得纳 米铜颗粒紧密地结合在三维石墨烯网格粉末上。然 后，进一步地在三维石墨烯网格粉末上包裹铜，形 成具有3D GN@Cu@Cu 结构的石墨烯/铜粉末, 最后热压成型, 如图 6 所示。实验结果表明此种工 艺获得的石墨烯增强铜基复合材料具有高强度和强 㓞性。

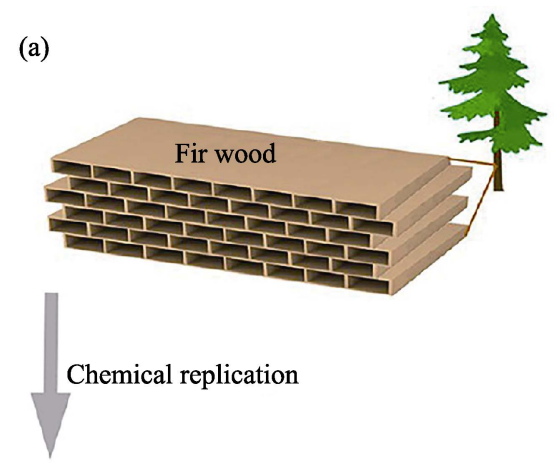

(b)

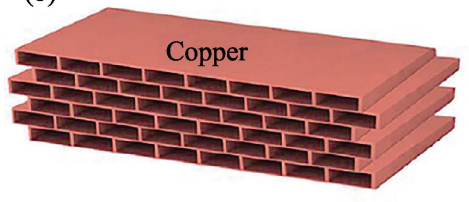

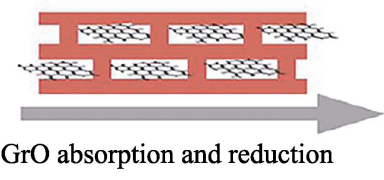

GrO absorption and reduction

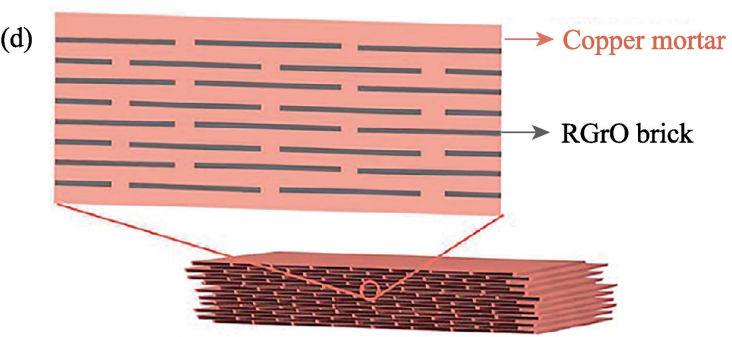

RGrO-and-copper nacre

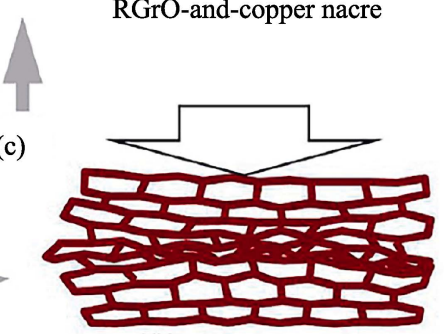

Hot-pressing

图 5 具有仿生贝壳结构的石墨烯铜基复合材料的制备过程示意图 ${ }^{[57]}$

Fig. 5 Schematic representation of fabricating RGrO-and-copper artificial nacre ${ }^{[57]}$

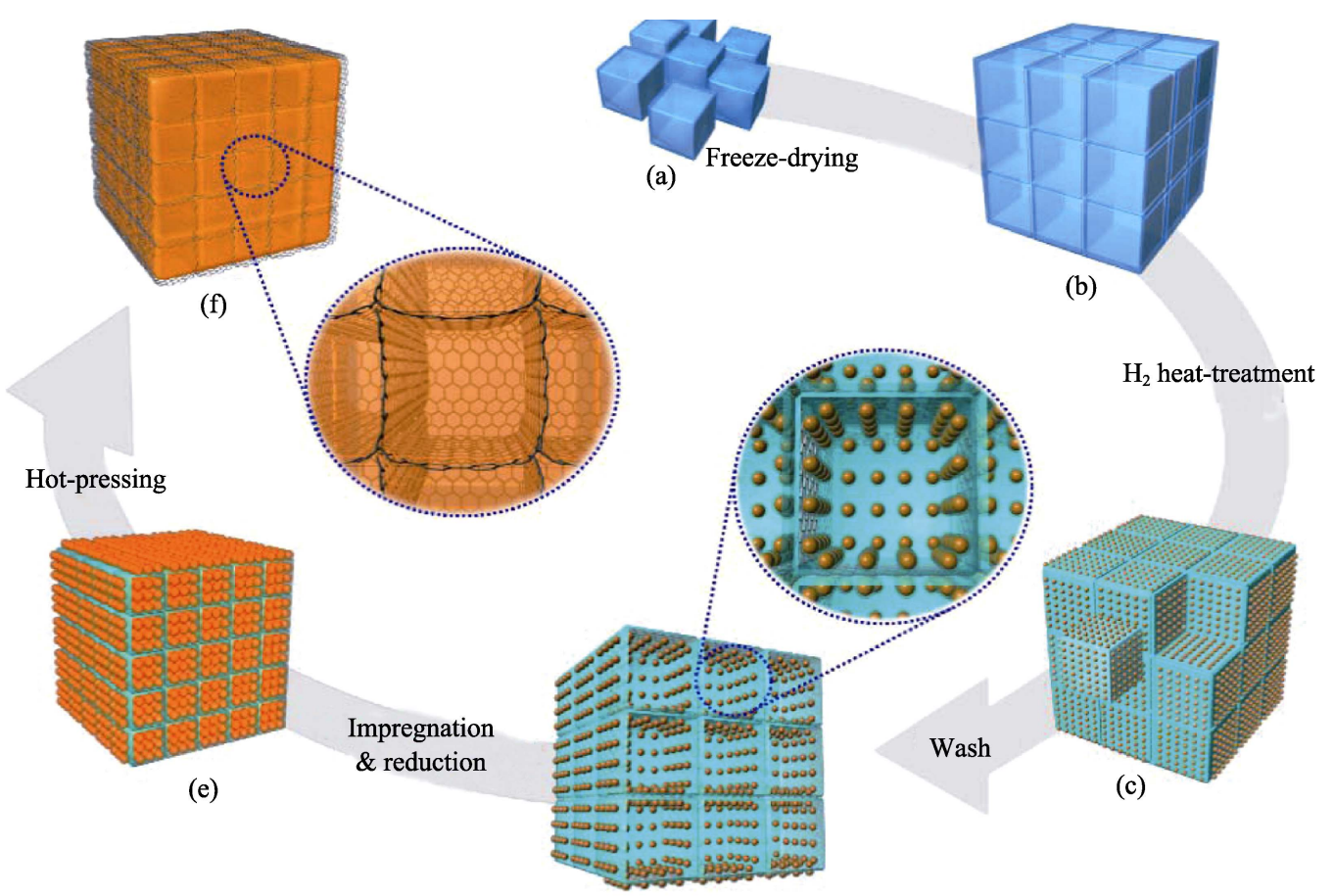

(d)

图 6 三维石墨烯网格/铜复合材料的制备过程示意图 ${ }^{[58]}$

Fig. 6 Schematic illustration of the overall production process for 3D GN/Cu composites ${ }^{[58]}$ 


\section{4 石墨烯增强机理}

大量实验研究验证了石墨烯对铜基材料力学性 能的增强作用，石墨烯增强铜基体的机制也引起了 广泛关注。研究人员定义强化效率 $R$ 以量化增强效 果, 增强相的增强效果与 $R$ 值成正比, 其表达式如 式(1) ${ }^{[28]}$ :

$$
R=\left(\sigma_{\mathrm{c}}-\sigma_{\mathrm{m}}\right) / V_{\mathrm{r}} \sigma_{\mathrm{m}}
$$

其中 $\sigma_{\mathrm{c}}$ 和 $\sigma_{\mathrm{m}}$ 分别代表复合材料的屈服应力和金属 基体的屈服应力, $V_{\mathrm{r}}$ 代表增强相的体积分数。Xiong 等 ${ }^{[57]}$ 通过预先成型浸渍工艺制备出石墨烯/铜珍珠 层状复合材料, 石墨烯的强化效率 $R$ 大约为 100 210 , 远远高于陶瓷颗粒 ${ }^{[59]}$ 、碳纤维 ${ }^{[5]}$ 、碳纳米管 ${ }^{[11]}$ 等增强相。石墨烯具有高 $R$ 值, 一方面在于石墨烯 的固有性质(优异的力学性能、独特的二维结构所拥 有的超高的比表面积), 另一方面是通过改进工艺 实现了石墨烯的均匀分布。复合材料一般由基体组 元与增强体或功能体组成, 利用组分之间的乘积效 应(协同作用)、系统效应、诱导效应、共轭效应等 来提高材料的性能 ${ }^{[60]}$ 。目前, 石墨烯增强铜基复合 材料的增强机制主要包括载荷转移、Orowan 强化、 细晶强化等 ${ }^{[60]}$ 。值得注意的是, 各种增强机制并不 是单一作用的，而是根据工艺条件的不同协同配合， 从而对基体达到增强效果。

载荷转移机制是当对复合材料施加应力时, 通 过石墨烯与铜基体的界面作用, 把载荷传递到强度 更高的石墨烯增强相上。因此, 铜基体与石墨烯之 间形成良好的界面结合力, 会大幅提升载荷转移的 能力, 从而增强基体的力学性能。在已有的研究中, 基于载荷转移机制的 Shear-lag 模型主要用于预测 短纤维增强金属基复合材料的力学性能 ${ }^{[61]}$ 。标准 Shear-lag 模型假设在基体和增强相的界面上没有发 生滑移, 纤维会重新分配材料中的应力, 同时载荷 将从基体转移到强度较大的纤维上 ${ }^{[62]}$ 。整个过程有 两个重要的推论: (1)在基体-纤维界面上存在剪切 应力; (2)增强相负载了大部分的应力, 而强度较弱 的基体承担了大部分的应变。近几年, 研究者发现 可以使用修正的 Shear-lag 模型预测石墨烯增强铜 基纳米复合材料的力学性能 ${ }^{[63]}$ 。根据修正的 Shear-lag 模型, 石墨烯复合材料的有效屈服强度可 以用式(2)表示:

$$
\sigma_{\mathrm{c}}=\sigma_{\mathrm{m}}(1+p V)
$$

其中 $\sigma_{\mathrm{m}}$ 是基体的屈服强度, $V$ 和 $p$ 分别是石墨烯的 体积分数和长宽比。由上述公式得出的理论数值与 实验数值几乎一致, 这一结果在验证载荷转移机制 存在的同时, 提供了一种预测石墨烯/金属复合材料 强度的方法。
Orowan 强化是指在复合材料受应力变形过程 中, 石墨烯对铜基体的物理分隔阻碍位错运动, 使 得位错在石墨烯附近堆积分布，阻止了位错的进一 步延伸，从而提高了材料的强度 ${ }^{[64]}$ 。这种强化过程 在纳米级别的复合材料中很容易观察到, 位错密度 是增强效率的主要影响因素。

细晶强化是指通过晶粒粒度的细化来提高金属 的强度, 多晶体金属的晶粒边界通常是大角度晶界, 相邻的不同取向的晶粒受力产生塑性变形时, 部分 施密特因子大的晶粒内位错源先开动, 并沿一定晶 面产生滑移和增殖 ${ }^{[63]}$ 。滑移至晶界前的位错被晶界 阻挡，这样一个晶粒的塑性变形就无法直接传播到 相邻的晶粒中去, 且造成塑变晶粒内位错塞积。目 前, 很多研究人员都意识到可以通过向金属或合金 基体中引入纳米级增强相实现晶粒细化，从而提升 基体的强度 ${ }^{[65]}$ 。晶粒越细, 晶界面积越大, 材料在 受力变形过程中, 滑移的位错在晶界处越容易被阻 挡，产生塞积。在铜基体中均匀分散的石墨烯纳米 片可以有效地钉扎晶界附近的位错, 造成位错堆 积。所以，在材料烧结成型过程中，石墨烯的存在可 以抑制晶粒长大, 有效地阻碍位错运动和裂纹扩 展。细晶强化的效果可以用 Hall-petch 公式 ${ }^{[60]}$ 描述, 晶粒的平均粒径越小, 材料的屈服强度就越高。此 外，石墨烯对于铜基体的增强机制还有热错配强化， 它主要是由于石墨烯与铜基体的热膨胀系数差异过 大所导致的。石墨烯的热膨胀系数约为 $10^{-6} \mathrm{~K}^{-1}$, 而 铜的膨胀系数为 $2.4 \times 10^{-5} \mathrm{~K}^{-1}$, 比石墨烯高一个数量 级。在烧结过程中, 两相的热变形差异会在铜基体 中产生高密度位错, 从而提高复合材料的强度 ${ }^{[66]}$ 。 但是, 当石墨烯含量过高时, 石墨烯将分布在晶界 处, 阻碍烧结过程中相邻颗粒间的结合, 从而降低 材料的致密化程度, 使材料的硬度下降。此外, 当石 墨烯含量增加到一定值后时，基体中单位体积内的 石墨烯片层数目增多，易于在层间范德华力作用下 团聚，使材料致密度降低，硬度下降 ${ }^{[64]}$ 。

对于铜基复合材料而言, 导电性能也是影响其 综合性能的关键。根据晶体理论 ${ }^{[67]}$, 材料内部电阻 的产生主要由于晶格完整性遭到破坏。石墨烯的加 入破坏了金属铜晶格体系的完整性, 使其出现严重 的晶格畸变, 这大幅增加了电子波的散射作用, 从 而使电阻增加，电导率下降。同时，石墨烯的弥散分 布阻碍了材料致密化, 使孔隙率增加、密度降低, 进 而使电导率降低。此外, 石墨烯的加入还有效阻碍 了晶粒长大，使晶界面积增大，同时也增加了其对 电子的散射作用，导致电导率下降。因此，如何合理 控制石墨烯在金属基体中的分布，保证复合材料的 
致密性是保证石墨烯增强铜基复合材料导电性能的 关键。

\section{3 结束语}

石墨烯作为铜基复合材料的增强相，对石墨烯/ 铜复合材料的力学性能提升明显。如何实现石墨烯 在铜基体中比较好的分散均匀性, 以及增强石墨烯 与铜基体的界面结合强度, 都会对复合材料的物理 性能产生较大影响。因此, 展望未来, 石墨烯增强铜 基复合材料的后续研究, 应重点关注以下几个方面:

1）通过工艺优化，精确控制石墨烯在铜基体 中的分散与分布形态, 消除团聚现象, 以避免复合 材料中生成“弱区”。同时, 深入研究石墨烯在铜基 体中的分布微观形貌对复合材料性能的影响。

2) 基于制备石墨烯的工艺路线不同，石墨烯 本身的层数、尺寸、缺陷程度相异, 其物理性能也 会大不相同。应针对不同复合材料的应用工况，选择 合适的石墨烯材料与铜基体复合，以达到优化性能的 目的。

3) 提高石墨烯与铜基体的界面结合强度, 有 效解决石墨烯与铜基体的润湿性问题, 进一步提高 复合材料的物理性能, 在提高复合材料力学性能的 同时，铜基体优异的导电、导热特性不会明显下降。

4) 致力于降低高温烧结或热压过程后复合材 料的孔隙率，提高致密度，这对复合材料的总体性 能有显著影响，目前文献较少有这方面的报导。

\section{参考文献:}

[1] RAJKOVIC V, BOZIC D, JOVANOVIC M T. Properties of copper matrix reinforced with various size and amount of $\mathrm{Al}_{2} \mathrm{O}_{3}$ particles. Journal of Materials Processing Tech., 2008, 200(1/2/3): 106-114.

[2] IBRAHIM I A, MOHAMED F A, LAVERNIA E J. Paniculate reinforced metal matrix composites-a review. Journal of Materials Science, 1991, 26(5): 1137-1156.

[3] IZMAILOV V V, USHAKOVA I N, DROZDOVA E I, et al. Electrical and tribological properties of composite material with copper matrix reinforced by superelastic hard carbon. Journal of Friction \& Wear, 2016, 37(3): 253-258.

[4] RAJKOVIC V, BOZIC D, JOVANOVIC M T. Properties of copper matrix reinforced with nano- and micro-sized $\mathrm{Al}_{2} \mathrm{O}_{3}$ particles. $M a$ terials Characterization, 2008, 459(1/2): 177-184.

[5] TENG L, CAIJU L I, YUAN Q, et al. Progress of carbon nanotubes reinforced copper matrix composites. Mater. Rev., 2014, 28(7): 16-19.

[6] BOZIC D, STASIC J, DIMCIC B, et al. Multiple strengthening mechanisms in nanoparticle-reinforced copper matrix composites. Bulletin of Materials Science, 2011, 34(2): 217-226.

[7] MCDANELS D L, JECH R W, WEETON J W. Stress-strain Behavior of Tungsten-fiber-reinforced Copper Composites. National Aeronautics And Space Administration Cleveland Oh Lewis Research Center, 1963.

[8] MAI Y J, CHEN F X, LIAN W Q, et al. Preparation and tribologi- cal behavior of copper matrix composites reinforced with nickel nanoparticles anchored graphene nanosheets. Journal of Alloys and Compounds, 2018, 756: 1-7.

[9] GUI C X, ZHAO X H, HUA X U, et al. Study and development of carbon fiber reinforced copper matrix coposites. Journal of Hebei University of Technology, 2002, 31(6): 43-48.

[10] XIA L, JIA B, ZENG J, et al. Wear and mechanical properties of carbon fiber reinforced copper alloy composites. Materials Characterization, 2009, 60(5): 363-369.

[11] BAKSHI S R, LAHIRI D, AGARWAL A. Carbon nanotube reinforced metal matrix composites - a review. Metallurgical Reviews, 2010, 55(1): 41-64.

[12] LIM B, KIM C, KIM B, et al. The effects of interfacial bonding on mechanical properties of single-walled carbon nanotube reinforced copper matrix nanocomposites. Nanotechnology, 2006, 17(23): $5759-5764$.

[13] NOVOSELOV K S, GEIM A K, MOROZOV S V, et al. Electric field effect in atomically thin carbon films. Science, 2004, 306(5696): 666-669.

[14] KAMALI A R, FRAY D J. Large-scale preparation of graphene by high temperature insertion of hydrogen into graphite. Nanoscale, 2015, 7(26): 11310-11320.

[15] SUN H, LI X, LI Y, et al. High-quality monolithic graphene films via laterally stitched growth and structural repair of isolated flakes for transparent electronics. Chemistry of Materials, 2017, 29(18): 7808-7815.

[16] VLASSIOUK I, SMIRNOV S, IVANOV I, et al. Electrical and thermal conductivity of low temperature CVD graphene: the effect of disorder. Nanotechnology, 2011, 22(27): 275716-1-9.

[17] KUMAR R, SINGH R K, DUBEY P K, et al. Pressure-dependent synthesis of high-quality few-layer graphene by plasma-enhanced arc discharge and their thermal stability. Journal of Nanoparticle Research, 2013, 15(9): 1847-1857.

[18] AKINWANDE D, BRENNAN C J, BUNCH J S, et al. A review on mechanics and mechanical properties of 2D materials - graphene and beyond. Extreme Mechanics Letters, 2017, 13: 42-77.

[19] DUTKIEWICZ J, OZGA P, MAZIARZ W, et al. Microstructure and properties of bulk copper matrix composites strengthened with various kinds of graphene nanoplatelets. Materials Science \& Engineering $A, 2015, \mathbf{6 2 8}$ : 124-134.

[20] WANG S, ZHANG Y, ABIDI N, et al. Wettability and surface free energy of graphene films. Langmuir, 2009, 25(18): 11078-11081.

[21] DAS S, LAHIRI D, LEE D Y, et al. Measurements of the adhesion energy of graphene to metallic substrates. Carbon, 2013, 59(7): 121-129.

[22] PONRAJ N V, AZHAGURAJAN A, VETTIVEL S C, et al. Graphene nanosheet as reinforcement agent in copper matrix composite by using powder metallurgy method. Surfaces \& Interfaces, 2017, 6: 190-196.

[23] LI JING-FU, ZHANG L, XIAO J K, et al. Sliding wear behavior of copper-based composites reinforced with graphene nanosheets and graphite. Transactions of Nonferrous Metals Society of China, 2015, 25(10): 3354-3362.

[24] RHO H, LEE S, BAE S, et al. Three-dimensional porous coppergraphene heterostructures with durability and high heat dissipation performance. Scientific Reports, 2015, 5: 12710-1-7.

[25] CHEN Y, ZHANG X, LIU E, et al. Fabrication of in-situ grown graphene reinforced $\mathrm{Cu}$ matrix composites. Scientific Reports, 2016, 6: 19363-1-9.

[26] PAVITHRA C L, SARADA B V, RAJULAPATI K V, et al. A new electrochemical approach for the synthesis of copper-graphene nanocomposite foils with high hardness. Sci. Rep., 2014, 4: 4049$1-7$.

[27] JAGANNADHAM K. Thermal conductivity of copper-graphene composite films synthesized by electrochemical deposition with exfoliated graphene platelets. Metallurgical and Materials Transactions B, 2011, 43(2): 316-324. 
[28] HWANG J, YOON T, JIN S H, et al. Enhanced mechanical properties of graphene/copper nanocomposites using a molecular-level mixing process. Adv. Mater., 2013, 25(46): 6724-6729.

[29] WANG L D, CUI Y, LI B, et al. High apparent strengthening efficiency for reduced graphene oxide in copper matrix composites produced by molecule-lever mixing and high-shear mixing. RSC Advances, 2015, 5(63): 51193-51200.

[30] ZHAO C. Enhanced strength in reduced graphene oxide/nickel composites prepared by molecular-level mixing for structural applications. Applied Physics A, 2015, 118(2): 409-416.

[31] HAUSNER H H. Modern Developments in Powder Metallurgy. New York: Plenum Press, 1973.

[32] ZABIHI M, TOROGHINEJAD M R, SHAFYEI A. Application of powder metallurgy and hot rolling processes for manufacturing aluminum/alumina composite strips. Materials Science \& Engineering A Structural Materials Properties Microstructure \& Processing, 2013, 560: 567-574.

[33] ELSAYED A, UMEDA J, KONDOH K. The production of powder metallurgy hot extruded Mg-Al-Mn-Ca alloy with high strength and limited anisotropy. Magnesium Technology, 2011, 2016: 475480.

[34] KOZBIAL A, LI Z, CONAWAY C, et al. Study on the surface energy of graphene by contact angle measurements. Langmuir, 2014, 30(28): 8598-8606.

[35] SEO J, CHANG W S, KIM T S. Adhesion improvement of graphene/ copper interface using UV/ozone treatments. Thin Solid Films, 2015, 584: 170-175.

[36] GAO X, YUE H, GUO E, et al. Mechanical properties and thermal conductivity of graphene reinforced copper matrix composites. Powder Technology, 2016, 301: 601-607.

[37] CHU K, JIA C. Enhanced strength in bulk graphene-copper composites. Physica Status Solidi, 2014, 211(1): 184-190.

[38] LI Z, WU P, WANG C, et al. Low-temperature growth of graphene by chemical vapor deposition using solid and liquid carbon sources. ACS Nano, 2011, 5(4): 3385-3390.

[39] SUN Z, YAN Z, YAO J, et al. Growth of graphene from solid carbon sources. Nature, 2010, 468(7323): 549-552.

[40] WU T, LIU Z, CHEN G, et al. A study of the growth-time effect on graphene layer number based on a $\mathrm{Cu}-\mathrm{Ni}$ bilayer catalyst system. RSC Advances, 2016, 6(28): 23956-23960.

[41] NEGISHI R, HIRANO H, OHNO Y, et al. Thickness control of graphene overlayer via layer-by-layer growth on graphene templates by chemical vapor deposition. Japanese Journal of Applied Physics, 2011, 50: 1271-1295.

[42] ISMACH A, DRUZGALSKI C, PENWELL S, et al. Direct Chemical Vapor Deposition of Single and Few--graphene Layers on Dielectric Surfaces. APS Meeting Abstracts. 2010.

[43] QU D, LI F Z, ZHANG H B, et al. Preparation of graphene nanosheets/copper composite by spark plasma sintering. Advanced Materials Research, 2014, 833: 276-279.

[44] LIU X, WEI D, ZHUANG L, et al. Fabrication of high-strength graphene nanosheets/Cu composites by accumulative roll bonding. Materials Science \& Engineering A, 2015, 642: 1-6.

[45] SAITO Y, UTSUNOMIYA H, TSUJI N, et al. Novel ultra-high straining process for bulk materials - development of the accumulative roll-bonding (ARB) process. Acta Materialia, 1999, 47(2): 579-583.

[46] HU Z, TONG G, LIN D, et al. Graphene-reinforced metal matrix nanocomposites-a review. Materials Science and Technology, 2016, 32(9): 930-953.

[47] KIM W, RIIKONEN J, ARPIAINEN S, et al. Growth of CVD graphene on copper by rapid thermal processing. MRS Proceedings, 2012, 1451: 27-32.

[48] KIM W J, LEE T J, HAN S H. Multi-layer graphene/copper composites: preparation using high-ratio differential speed rolling, microstructure and mechanical properties. Carbon, 2014, 69(4): $55-65$.
[49] PAVITHRA C L P, SARADA B V, RAJULAPATI K V, et al. Process optimization for pulse reverse electrodeposition of graphenereinforced copper nanocomposites. Advanced Manufacturing Processes, 2015, 31(11): 1439-1446.

[50] ZHANG D, ZHAN Z. Strengthening effect of graphene derivatives in copper matrix composites. Journal of Alloys \& Compounds, 2016, 654: 226-233.

[51] BARTOLUCCI S F, PARAS J, RAFIEE M A, et al. Graphenealuminum nanocomposites. Materials Science \& Engineering A, 2011, 528(27): 7933-7937.

[52] ZHU W, CHANG Q, CHEN L, et al. Preparation and properties of reduced graphene oxide reinforced copper matrix composites. Journal of Wuhan University of Science \& Technology, 2018, 41(1): 37-43.

[53] PENG Y, HU Y, HAN L, et al. Ultrasound-assisted fabrication of dispersed two-dimensional copper/reduced graphene oxide nanosheets nanocomposites. Composites Part B, 2014, 58(3): 473-477.

[54] CHEN X, TAO J, YI J, et al. Strengthening behavior of carbon nanotube-graphene hybrid in copper matrix composite. Materials Science \& Engineering A, 2018, 718: 427-436.

[55] CUI YE, WANG LIDONG, CAO GUOJIAN, et al. Effect of ball milling on the defeat of few-layer graphene and properties of copper matrix composites. Acta Metallurgica Sinica, 2014, 27(5): 937-943.

[56] LI M X, XIE J, LI Y D, et al. Reduced graphene oxide dispersed in copper matrix composites: facile preparation and enhanced mechanical properties. Physica Status Solidi, 2015, 212(10): 2154 2161.

[57] XIONG D B, CAO M, GUO Q, et al. Graphene-and-copper artificial nacre fabricated by a preform impregnation process: bioinspired strategy for strengthening-toughening of metal matrix composite. ACS Nano, 2015, 9(7): 6934-6943.

[58] ZHANG X, SHI C, LIU E, et al. Achieving high strength and high ductility in metal matrix composites reinforced with a discontinuous three-dimensional graphene-like network. Nanoscale, 2017, 9(33): 11929-11938.

[59] TU J, WANG N, YANG Y, et al. Preparation and properties of $\mathrm{TiB}_{2}$ nanoparticle reinforced copper matrix composites by in situ processing. Materials Letters, 2002, 52(6): 448-452.

[60] BAIG Z, MAMAT O, MUSTAPHA M. Recent progress on the dispersion and the strengthening effect of carbon nanotubes and graphene-reinforced metal nanocomposites: a review. Critical Reviews in Solid State and Materials Sciences, 2018, 43(1): 1-46.

[61] TAYA M, ARSENAULT R. A comparison between a shear lag type model and an eshelby type model in predicting the mechanical properties of a short fiber composite. Scripta Metallurgica, 1987, 21(3): 349-354.

[62] ZHAO P, JI S. Refinements of shear-lag model and its applications. Tectonophysics, 1997, 279(1-4): 37-53.

[63] TANG Y, YANG X, WANG R, et al. Enhancement of the mechanical properties of graphene-copper composites with graphenenickel hybrids. Materials Science and Engineering: A, 2014, 599: 247-254.

[64] TJONG S C. Recent progress in the development and properties of novel metal matrix nanocomposites reinforced with carbon nanotubes and graphene nanosheets. Materials Science and Engineering: R: Reports, 2013, 74(10): 281-350.

[65] ZHANG D, ZHAN Z. Preparation of graphene nanoplateletscopper composites by a modified semi-powder method and their mechanical properties. Journal of Alloys and Compounds, 2016, 658: $663-671$

[66] MILLER W S, HUMPHREYS F J. Strengthening mechanisms in particulate metal matrix composites. Scripta Metallurgica et Materialia, 1991, 25(1): 33-38.

[67] MOCKO, J L SARRAO et al. Microhardness of the $\mathrm{Yb}_{x} \mathrm{Y}_{1-x} \mathrm{InCu}_{4}$ alloy system: the influence of electronic structure on hardness. Journal of Physics: Condensed Matter, 15(50): 8719-8723. 